\title{
CARACTERIZACIÓN MOLECULAR DE CLONES DE Theobroma cacao L., POR MEDIO DE MARCADORES MOLECULARES MICROSATÉLITES
}

\author{
JULIANA LONDOÑO MURILLO ${ }^{1}$, DIANA MARÍA GIL VILLA, SANDRA BIBIANA AGUILAR MARÍN², \\ FREDY ARVEY RIVERA PÁEZ ${ }^{3}$ Y GERMÁN ARIEL LÓPEZ GARTNER ${ }^{4}$ \\ german.lopez@ucaldas.edu.co
}

Manizales, 2010-11-11 (Rev. 2011-03-09)

\section{RESUMEN}

Theobroma cacao L. es la única especie del género Theobroma que se explota comercialmente en grandes extensiones, registrando en la actualidad una amplia distribución mundial, a través de programas de desarrollo directamente influenciados por factores vinculados al mercado y por los intereses de productores, comerciantes, industriales y consumidores. En este estudio se evaluaron 12 clones de cacao por medio de marcadores moleculares utilizando 10 secuencias microsatélites (SSRs), como un estudio piloto antes de un ensayo a mayor escala. Los datos se procesaron mediante el programa Power Marker Versión 3.25. Se estimaron las frecuencias alélicas y luego se generó una matriz de distancias genéticas basada en el coeficiente de Nei. Utilizando el algoritmo de agrupamiento UPGMA se generó el dendrograma correspondiente. El análisis de diversidad mostró un índice de diversidad genética total de 0,6944 considerado intermedio para los materiales evaluados. La estimación de la heterocigosidad promedio fue de 0,58579 y de promedio del contenido de información polimórfica de 0,6523. En este estudio los marcadores mTcCIR6, mTcCIR25, mTcCIR26 y mTcCIR12 son los más informativos y polimórficos. Se recomienda relacionar los resultados de diversidad genética con caracteres morfo-agronómicos y de patogenicidad en los distintos clones de cacao a fin de consolidar estrategias eficaces de mejoramiento y control de enfermedades.

\section{PALABRAS CLAVE:}

Theobroma cacao L., microsatélites, diversidad genética, fitomejoramiento, germoplasma.

\section{Theobroma cacao L. CLONES MOLECULAR CHARACTERIZATION USING MOLECULAR MICROSATELLITE MARKERS}

\section{ABSTRACT}

Theobroma cacao L. is the only Theobroma species that is commercially exploited in enormous extensions, registering presently wide distribution worldwide through development programs directly influenced by factors linked to the market and the producers, traders, industrials, and consumers' interests. In this study 12 cacao clones were evaluated by means of molecular markers using 10 microsatellite sequences (SSRs) as a pilot study before a test at a greater scale. The data were processed using the Power Maker Version 3.25 program. The allelic frequencies were stimated and a genetic distance matrix was developed based on the Nei coefficient. Using the UPGMA grouping algorithm the corresponding dendrogram was generated. The diversity analysis showed a total genetic diversity index of 0.6944 considered intermediate for the materials evaluated. The average heterozygocity estimate was 0.58579 and the average polymorphic information content was 0.6523 . In this study markers mTcCIR6, mTcCIR25, mTcCIR26 and mTcCIR12 are the most informative and polymorphic ones. It is recommended to relate the genetic diversity results with morpho-agronomic and pathogenicity characters in the different cacao clones in order to consolidate efficient strategies for disease improvement and control.

\section{Key Words:}

Theobroma cacao L., microsatellites, genetic diversity, phyto improvement, germoplasm.

\section{INTRODUCCIÓN}

El cacao, Theobroma cacao L., es una especie de origen neotropical que se desarrolla entre los $18^{\circ}$ de latitud norte y los $15^{\circ}$ de latitud sur (Cuatrecasas, 1964 citado por Girón, Tortolero y Sánchez., 2007). Pertenece a la familia Sterculiaceae y su crecimiento está restringido a biotopos de zonas de alta pluviosidad, donde las plantas se desarrollan asociadas a bosques naturales de manera 
permanente. Además, es la única especie del género Theobroma que se explota comercialmente en grandes extensiones. Registra en la actualidad una amplia distribución mundial, lo cual ha sido posible gracias a diversos programas de desarrollo y mercadeo, asociados a la demanda creciente de los numerosos productos derivados del cacao (Girón et al., 2007).

Los principales productores son los países africanos. Costa de Marfil concentra el $40 \%$ de la producción mundial, Ghana y Nigeria participan con el $13 \%$ y $10 \%$, respectivamente. Colombia se ubica como noveno productor mundial después de Brasil, Camerún, Ecuador y Malasia, con una participación pequeña de 1,5\% (Agrocadenas, 2005).

En Colombia, el cultivo de cacao se desarrolló con base en materiales genéticos híbridos obtenidos por cruzamientos entre clones trinitarios y amazónicos y, en buena proporción, hijos de híbridos; por ello cobra gran importancia el estudio genético de los clones en relación con su potencial de uso para dirigir los cruzamientos en los programas de mejoramiento. Además, es muy bajo el porcentaje del área sembrada que está establecido con materiales genéticos provenientes de clones extranjeros. De acuerdo con la Compañía Nacional de Chocolates, el 30\% de la superficie cacaotera está sembrada con materiales comunes, el $67 \%$ con híbridos y solo el $3 \%$ con clones. Actualmente, se están produciendo plántulas clonadas de materiales élite, que han demostrado resistencia a enfermedades y productividades entre 1 y 2 ton/ha (Agrocadenas, 2005).

La caracterización de la biodiversidad de los recursos fitogenéticos está considerada entre las líneas de investigación estratégicas a nivel mundial, debido a que se perfila como la estrategia fundamental para la solución de los problemas actuales de los cultivos, a través del mejoramiento genético asistido por marcadores moleculares, la adaptación a los cambios climáticos y el desarrollo de nuevas alternativas de producción (Virk, Ford-Lloyd, Jackson y Newbury, 1995).

En los últimos años, con el advenimiento de técnicas basadas en la manipulación del ADN, se ha desarrollado una nueva generación de descriptores, los marcadores moleculares, que permiten una identificación genotípica precisa; se denomina huellas dactilares de ADN "DNA finger printing" (Tolares, Marcucci y Harrond, 2005).

Los marcadores moleculares se caracterizan por estar ampliamente distribuidos en todo el genoma, tener herencia mendeliana y no estar influenciados por el medio ambiente, por lo cual resultan adecuados para su utilización en la identificación varietal de especies y la caracterización de la diversidad genética. Los microsatélites (Single Sequence Repeats, SSRS) son marcadores moleculares altamente polimórficos, y su característica de ser codominantes los potencia como una herramienta muy poderosa para generar una identificación confiable de organismos y permitir una evaluación adecuada de la diversidad genética (Tolares et al., 2005).

En las variedades de cacao, los estudios de diversidad utilizando marcadores moleculares son más bien escasos. Quiroz (2002) realizó una caracterización molecular de 63 genotipos de cacao procedentes de Costa Rica y una evaluación morfológica de 51 clones de la estación experimental de Pichilingue (Ecuador), además de tres genotipos de referencia de los tipos genéticos trinitario (UF-676), forastero amazónico (Matina) y criollo (Criollo-36); el dendrograma para los 63 clones de cacao, demostró la no-conformación de grupos genéticos específicos, pero sí separados por su origen y constitución genética. En otro estudio Cryer, Fenn, Tumbull y Wilkinson (2006) realizaron el primer esfuerzo para la sistematización de microsatélites a nivel internacional. Palacio (2007) realizó una caracterización molecular de Theobroma cacao L., con marcadores moleculares AFLP, en la que obtuvo un total de 250 marcadores que fueron evaluados en 13 clones de interés comercial provenientes de una zona cafetera marginal de Colombia. Marcano, Morales, Hoyer, Courtois y Risterucci (2009) utilizaron la técnica de mapeo genético analizando características importantes para el cultivo del cacao, además, se detectó el grado de variación o polimorfismo en las muestras fenotípicamente distintas, así como la formación de dos grupos genéticos, el primero incluyó clones de origen trinitario y el segundo clones de tipo trinitario y forastero.

En este trabajo se realiza un análisis molecular preliminar de la diversidad genética de 12 clones de Theobroma cacao L. de importancia económica, mediante el uso de marcadores moleculares microsatélites (SSRs), en procura de un primer acercamiento a la caracterización de la diversidad genética de materiales con valor agronómico para los programas de fitomejoramiento de los cultivares de cacao de nuestra región y del país.

\section{MATERIALES Y MÉTODOS}


La colecta del material vegetal se realizó en la Granja Luker ubicada en la región de Santágueda, del municipio de Palestina (Caldas). Tiene una extensión de 22,5 hectáreas y se ubica a 1050 $\mathrm{msnm}$. Presenta una precipitación promedio anual de $2200 \mathrm{~mm}$ de lluvia muy bien distribuidos, brillo solar de 2.200 horas y temperatura promedio de $23^{\circ} \mathrm{C}$ (Casa Luker, 2007). De cada planta se colectaron hojas jóvenes y en buen estado fitosanitario que fueron envueltas en papel aluminio, rotuladas y almacenadas en una nevera de icopor con hielo hasta su respectivo procesamiento en el Laboratorio de Genética de la Universidad de Caldas, con el fin de evitar la fenolización de las hojas. Se maceró en un mortero con nitrógeno líquido aproximadamente $1 \mathrm{~g}$ de hojas jóvenes. El material sobrante se almacenó como respaldo a $-20^{\circ} \mathrm{C}$.

Los clones evaluados fueron: seis materiales de origen trinitario: CCN51, ICS1, ICS39, ICS60, ICS95 y TSH565; y seis materiales de origen forastero: EET8, IMC67, Luker21, Luker40, PA150 y $\mathrm{P} 7$, seleccionados según criterios de productividad.

Para la extracción de ADN se usó el protocolo descrito por Doyle y Doyle (1990) y Doyle (1991), modificado por Márquez (2003). El tampón de extracción incluye CTAB y $\beta$-mercaptoetanol, y la purificación de ADN se realizó con cloroformo-alcohol isoamílico. La calidad y cantidad del ADN total extraído se evaluó en geles de agarosa al $0,8 \%$ teñidos con bromuro de etidio y visualizados en luz ultravioleta. La estimación de la concentración aproximada de ADN se hizo por comparación con un estándar. El ADN se almacenó a $-20^{\circ} \mathrm{C}$ hasta su utilización.

Para la amplificación de los microsatélites por PCR se utilizó el protocolo de estandarización de perfiles térmicos de McCouch (2002). La optimización de perfiles para PCR se desarrolló así: desnaturalización inicial $96^{\circ} \mathrm{C}$ por 2 minutos, seguido por 35 ciclos de desnaturalización $96^{\circ} \mathrm{C}$ por 1 minuto, acoplamiento $46 \circ 51^{\circ} \mathrm{C}$ (según el iniciador) por 1 minuto y un tiempo de elongación $72^{\circ} \mathrm{C}$ por 2 minutos. Una extensión final $72^{\circ} \mathrm{C}$ por 5 minutos y enfriamiento a $10^{\circ} \mathrm{C}$ por 10 minutos. Conservación a $4^{\circ} \mathrm{C}$ hasta evaluación electroforética.

Se utilizaron 12 secuencias de iniciadores específicos para genoma nuclear de materiales de cacao adaptados de Cryer et al. (2006), y seleccionados por su valor de heterocigosidad según estos autores (Tabla 1).

Tabla 1. Secuencias de marcadores moleculares microsatélites. Secuencias de 12 iniciadores de PCR para microsatélites de genoma nuclear de Theobroma cacao L. utilizados para el análisis de diversidad.

\begin{tabular}{|l|l|}
\hline Marcador & Secuencia de los iniciadores $\left(5^{\prime} \rightarrow \mathbf{3}^{\prime}\right)$ \\
\hline mTcCIR6 & $\begin{array}{l}\text { TCCCTCTAAACTACCCTAAAT } \\
\text { TAAAGCAAAGCAATCTAACATA }\end{array}$ \\
\hline mTcCIR7 & $\begin{array}{l}\text { ATGCGAATGACAACTGGT } \\
\text { GCTTTCAGTCCTTTGCTT }\end{array}$ \\
\hline mTcCIR8 & $\begin{array}{l}\text { CTAGTTCCCATTACCA } \\
\text { TCCTCAGCATTTCTTTC }\end{array}$ \\
\hline mTcCIR11 & $\begin{array}{l}\text { TTGGTGATTATAGGCAG } \\
\text { GATCGATTGATGTGAG }\end{array}$ \\
\hline mTcCIR12 & $\begin{array}{l}\text { TCTGACCCCAAACCTGTA } \\
\text { ATTCCAGTTAAAGCACAT }\end{array}$ \\
\hline mTCCIR18 & $\begin{array}{l}\text { GATAGCTAAGGGGTTGAGGA } \\
\text { GGTAATCAATCATTGAGGATA }\end{array}$ \\
\hline mTCCIR25 & $\begin{array}{l}\text { CTCGTAGTGAATGTAGGAG } \\
\text { TAGGTAGGTAGGGTATCT }\end{array}$ \\
\hline mTcCIR26 & $\begin{array}{l}\text { GCATCATCATACATTC } \\
\text { GCACTCAAAGTCATACTAC }\end{array}$ \\
\hline mTCCIR33 & $\begin{array}{l}\text { TGGGTGAAGATTGGT } \\
\text { CAACAATGAAATAGGCA }\end{array}$ \\
\hline mTCCIR37 & $\begin{array}{l}\text { CTGGGTGCTGATAGATAAT } \\
\text { ATTTGTGGGAGGGTAT }\end{array}$ \\
\hline mTCCIR40 & $\begin{array}{l}\text { AATCCGACAGTCTTAATC } \\
\text { CTTAAATGTTATGTGTATGC }\end{array}$ \\
\hline mTCCIR60 & $\begin{array}{l}\text { CTTAAATGTTATGTGTATGC } \\
\text { AGAGCAACCATCACTAATCA }\end{array}$ \\
\hline
\end{tabular}


Los productos del amplificación se separaron por electroforesis en geles desnaturalizantes de poliacrilamida al $4 \%$, y se observaron mediante tinción con nitrato de plata según el protocolo adaptado de McCouch (2002).

Análisis estadístico

Los datos de genotipos se procesaron mediante el paquete estadístico Power Marker Versión 3.25 (Liu y Muse, 2005), con el cual se estimaron los parámetros genéticos. Las distancias fenéticas permitieron generar una matriz de distancias entre las unidades taxonómicas operativas (UTO) calculadas a partir de los patrones de bandas. Las distancias se definen como 1-S, siendo $S$ el coeficiente de similitud; el cual se estimó por el coeficiente de Nei:

$$
S_{i j}=\frac{2 a}{(2 a+b+c)}
$$

Donde, Sij es la similitud entre las UTO $i$ y $j$; a es el número de bandas presentes en ambas UTO $i$ y $j$; b es el número de bandas presentes en $i$ y ausentes en $j$, y $c$ es el número de bandas presentes en $j$ y ausentes en $i$. La distancia fenética para la construcción de la matriz de distancias y el dendrograma se define como 1-Sij. Para la construcción del dendrograma se realizó el análisis de agrupamiento aplicando el algoritmo UPGMA (ligamiento promedio utilizando la media aritmética no ponderada) (Sneath y Sokal, 1973). Las frecuencias alélicas se estimaron utilizando la expresión:

$$
\tilde{p}_{u}=\frac{n_{u}}{2 n}
$$

Donde $n u$ representa los conteos totales de alelos de tipo $u$ y $2 n$ es el número total de alelos en un locus dado.

La diversidad genética se evaluó a partir de tres estimaciones: el número de alelos por locus $\left(A_{l}\right)$, la heterocigosidad $\left(\widehat{H}_{l}\right)$ y el índice de diversidad genética $\left(\widehat{D}_{l}\right)$, La estimación de la heterocigosidad para un locus dado es simplemente la proporción de individuos heterocigóticos en dicho locus:

$$
\widehat{H}_{l}=1-\sum_{u=1}^{k} \tilde{P}_{l u u}
$$

Donde $\tilde{P}_{l u u}$ es la frecuencia genotípica observada de individuos homocigóticos uu en el locus / y la sumatoria se hace a lo largo de todos los alelos del mismo locus.

La diversidad genética, también referida como heterocigosidad esperada, se define como la probabilidad de que dos alelos de un mismo locus escogidos al azar sean diferentes:

$$
\widehat{D}_{l}=\left(1-\sum_{u=1}^{k} \tilde{p}_{l u}^{2}\right)
$$

Donde $\tilde{p}_{l u}^{2}$ es la frecuencia alélica observada del alelo $u$ en el locus / y la sumatoria se hace a lo largo de todos los alelos del mismo locus.

El contenido de información de polimorfismo o $\overline{P \overline{I C}} l$ (Polymorphism Information Content) es una estimación de diversidad que permite clasificar los marcadores genéticos según su nivel de polimorfismo (Botstein, White, Skolnick y Davis, 1980). Se estimó a partir de la expresión: 


$$
\widehat{P I C_{l}}=1-\sum_{u=1}^{k} \tilde{p}_{l u}^{2}-\sum_{u=1}^{k-1} \sum_{v=u+1}^{k} 2 \tilde{p}_{l u}^{2} \tilde{p}_{l v}^{2}
$$

\section{RESULTADOS}

Algunos materiales de cacao resultaron ser recalcitrantes para la extracción de ADN, en particular, al material Luker21 no fue posible hacerle una extracción de buena calidad, por lo cual se necesitó trabajar con muestras frescas y extremar las medidas de control de fenolización del tejido vegetal. En cuanto a los marcadores, dos de ellos no permitieron obtener amplificaciones legibles en los geles, por lo cual hubo que prescindir de ellos. Los resultados finales de este estudio incluyen 11 materiales de cacao analizados genéticamente mediante los 10 mejores marcadores microsatélites en términos de amplificación y polimorfismo. Un ejemplo de marcador polimórfico se presenta en la Figura 1.

Figura 1. Amplificación del gen marcador mTcCIR25.

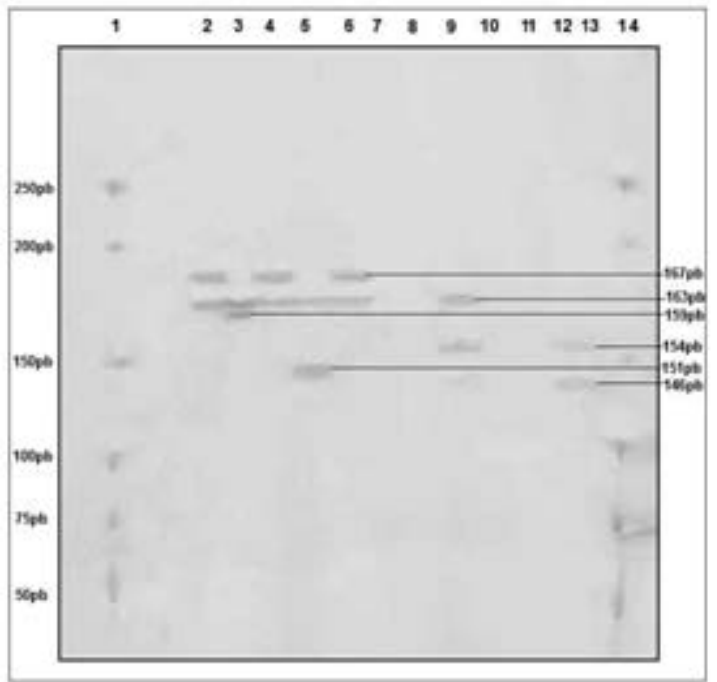

Gel desnaturalizante de poliacrilamida al 4\%. Carriles numerados de izquierda a derecha: 1, marcador de peso molecular (25 pb); 2, ICS60; 3, ICS1; 4, ICS95; 5, TSH565; 6, EET8; 7, ICS39; 8, CCN51; 9, Luker40; 10, Luker21; 11, IMC67; 12, P7; 13, PA150; 14, marcador de peso molecular $(25 \mathrm{pb})$.

Las estimaciones de diversidad genética se realizaron para 11 materiales de cacao utilizando 10 marcadores microsatélites. Estas incluyeron el número de alelos por locus, la heterocigosidad por locus, la diversidad genética o heterocigosidad esperada y el contenido de información polimórfica. Los datos se presentan en la Tabla 2.

Tabla 2. Estimaciones de la diversidad genética. Se calcularon tres estimadores de diversidad para los 10 mejores marcadores evaluados en 11 clones de Theobroma cacao L. de la Granja Luker: $A_{l}$ , número de alelos por locus; $\widehat{H}_{l}$, heterocigosidad por locus; $\widehat{D}_{l}$, diversidad genética o heterocigosidad esperada; y $\vec{P} \widehat{I C} l$ contenido de información polimórfica. 


\begin{tabular}{|l|l|l|l|c|}
\hline Marcador & $\boldsymbol{A}_{l}$ & \multicolumn{1}{|c|}{$\tilde{\boldsymbol{H}}_{l}$} & \multicolumn{1}{|c|}{$\tilde{\boldsymbol{D}}_{l}$} & $\widetilde{\boldsymbol{P I C}} \boldsymbol{C}_{l}$ \\
\hline mTcCIR6 & 8 & 0,8179 & 0,8611 & 0,8468 \\
\hline mTcCIR7 & 3 & 0,5400 & 0,6527 & 0,5994 \\
\hline mTcClR8 & 3 & 0,5935 & 0,5833 & 0,5295 \\
\hline mTcCIR11 & 3 & 0,3950 & 0,5833 & 0,5295 \\
\hline mTcCIR12 & 5 & 0,7671 & 0,7638 & 0,7393 \\
\hline mTcCIR18 & 3 & 0,5221 & 0,6250 & 0,5593 \\
\hline mTcCIR25 & 8 & 0,5178 & 0,8194 & 0,7956 \\
\hline mTcCIR26 & 6 & 0,7750 & 0,8050 & 0,7818 \\
\hline mTcCIR33 & 2 & 0,2135 & 0,4861 & 0,4235 \\
\hline mTcClR40 & 4 & 0,7160 & 0,5416 & 0,4598 \\
\hline Promedio & $\mathbf{0 , 5 8 5 7 9}$ & $\mathbf{0 , 6 9 4 4}$ & $\mathbf{0 , 6 5 2 3}$ \\
\hline
\end{tabular}

El análisis de agrupamiento también incluye los 11 materiales que permitieron una mejor calidad del ADN, y los 10 marcadores microsatélites que arrojaron las mejores amplificaciones. Los resultados se presentan en la Figura 2.

Figura 2. Dendrograma de agrupamiento de los materiales de cacao.

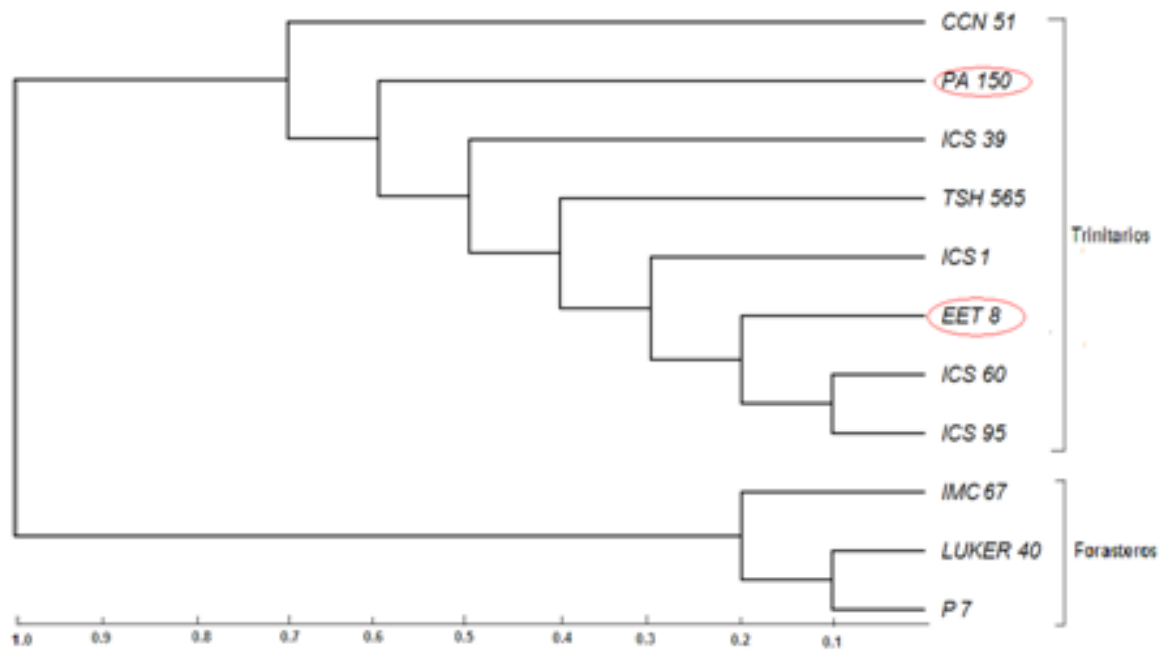

Se utilizaron 10 marcadores microsatélites aplicados a 11 clones de Theobroma cacao L. El dendrograma se construyó utilizando la distancia entre UTO (1-S) a partir del coeficiente de similitud de Nei y el método de agrupamiento UPGMA. A la derecha se indica el origen de los materiales (trinitarios y forasteros) y en óvalos dos materiales forasteros que agruparon con el conjunto de los trinitarios.

\section{DISCUSIÓN}

Este estudio piloto, en el cual se evaluaron once clones de Theobroma cacao L. utilizando diez marcadores moleculares microsatélites, sugiere que, a pesar de tratarse de materiales propagados clonalmente y de utilización comercial, en general existe un nivel importante de diversidad genética en los materiales de cacao conservados en la Granja Luker, diversidad que supone un potencial valioso que puede ser explotado favorablemente en beneficio del fitomejoramiento de este cultivo. 
Se encontró que el cacao es recalcitrante a la extracción de ADN por tener una alta tendencia a la fenolización de las muestras, ante esto se recomienda la utilización de columnas de purificación para garantizar una óptima calidad en los resultados de amplificación de microsatélites por PCR.

Los marcadores mTcCIR6 y mTcCIR25 mostraron mayor polimorfismo con un total de ocho alelos. Los marcadores mTcCIR12, mTcCIR26 y mTcCIR40 mostraron seis, cinco y cuatro alelos, respectivamente. Los marcadores $\mathrm{mTcCIR} 7, \mathrm{mTcCIR} 8, \mathrm{mTcCIR} 11$ y $\mathrm{mTcCIR} 18$ presentaron tres alelos cada uno, y el iniciador con menor número de alelos fue el mTcCIR33 con solamente dos. Estos datos sugieren que, para el número limitado de muestras evaluadas, existe un nivel de polimorfismo importante en estos marcadores genéticos, expresado como número de alelos por locus.

Todas las estimaciones de diversidad genética realizadas, incluyendo número de alelos por locus, heterocigosidad por locus, diversidad y contenido de información polimórfica, indican que los marcadores mTcCIR6, mTcCIR12, mTcCIR25 y mTcCIR26, presentan la mayor variabilidad entre los clones evaluados, y por lo tanto, un mayor poder discriminante. Estos resultados concuerdan con lo hallado por Cryer et al. (2006), quienes registran que estos marcadores presentan la mayor cantidad de alelos y se constituyen como altamente polimórficos y representativos para la especie.

Por esta razón, estos marcadores deberían incluirse prioritariamente en los estudios de caracterización, definición de origen probable y, en general, para la evaluación de la diversidad genética en los diferentes materiales de cacao, especialmente si se planea abordar un estudio a mayor escala con un mayor número de muestras de diferentes localidades, o si se piensa en conformar una colección nuclear (core collection) que represente la mayor diversidad genética posible con el menor número de materiales, a fin de reducir los costos de mantenimiento.

En cuanto a la estructura genética que reveló el análisis de agrupamiento, se puede apreciar que el análisis del dendrograma permite separar los materiales evaluados en dos grupos principales, uno de ellos compuesto por seis materiales de procedencia trinitaria (CCN51, ICS39, TSH565, ICS1, ICS60 e ICS95) y dos materiales forasteros (EET8 y PA150), que presentan orígenes híbridos entre forasteros y trinitarios, lo cual explica esta tendencia de agrupación. El otro grupo, se compone de tres materiales forasteros exclusivamente (IMC67, Luker40 y P7). La asociación de los clones con su sitio de origen concuerda con lo reportado por Quiroz (2002) y Marcano et al. (2009). Esto sugiere que es posible utilizar los marcadores más polimórficos de este estudio, complementándolos con algunos adicionales, con el fin de desarrollar una herramienta molecular que ayude en el diagnóstico de origen probable de los materiales de cacao o de algunos de sus productos derivados. En el mismo sentido, es posible también utilizar esta estrategia para evaluar el grado de introgresión genética de los materiales híbridos que se obtienen durante los programas de mejoramiento.

Cuando se analizaron los resultados de los patrones de bandas detalladamente, se encontró que los materiales forasteros que agruparon juntos en el dendrograma, Luker40, P7 e IMC67, difieren entre ellos en cuatro alelos. En el grupo conformado mayoritariamente por materiales trinitarios, se encontró que los materiales ICS60 e ICS95 presentan el mismo número de alelos con un perfil de bandeo casi idéntico, mientras que los materiales ICS1 e ICS39 difieren de los anteriores en tres y seis alelos respectivamente, mostrando que, a pesar de pertenecer a la variedad Imperial College Selection, existe gran diversidad genética dentro de esta colección que debe ser estudiada más ostensiblemente para ser aprovechada en programas de mejoramiento del cultivo de cacao.

En Colombia han sido insipientes los estudios de diversidad genética en cacao utilizando marcadores moleculares microsatélites, solo se registran dos trabajos en la especie para el país, por lo tanto se considera de gran importancia este análisis aportando un conocimiento preliminar a nivel molecular y de variabilidad genética para Theobroma cacao L., en Caldas. Además, se hace evidente y necesario que este tipo de investigaciones se integren a otras áreas como: mejoramiento genético, conservación, control biológico y así apuntar a desarrollo de programas para el fitomejoramiento de los cultivos.

\section{AGRADECIMIENTOS}

Al Grupo de Investigación GEBIOME (Genética, Biodiversidad y Fitomejoramiento), por su soporte logístico, académico, técnico y humano. A la Compañía Casa Luker y la Granja Luker, por permitirnos realizar muestreos de material vegetal en sus colecciones de germoplasma. A Cenicafé, por el entrenamiento recibido en aspectos técnicos en el Laboratorio de Marcadores Moleculares, dirigido por la Dra. Pilar Moncada y a la Vicerrectoría de Investigaciones y Postgrados de la Universidad de Caldas por la financiación de este proyecto con lo cual se promueve la investigación aplicada. 


\section{REFERENCIAS}

- Agrocadenas. (2005). La cadena de cacao en Colombia: Una mirada global de su estructura y dinámica. Documento de trabajo, 92. Ministerio de Agricultura y Desarrollo Rural. Observatorio de Agrocadenas Colombia. pp. 4-5.

- Botstein, D., White, R. L., Skolnick, M., y Davis, R. W. (1980). Construction of a genetic linkage map in man using restriction fragment length polymorphisms. American Journal of Human Genetics, 32, 314-331.

- Casa Luker. (2007). Granja Luker. http://www.casaluker.com/espanol/granjaluker.html

- Cryer, N. C., Fenn, M. G., Tumbull, C. J., y Wilkinson, M. J. (2006). Allelic size standars and reference genotypes to unify international cocoa (Theobroma cacao L.) microsatellite data. Genetic Resources and Crop Evolution, 53, 1643-1652.

- Cuatrecasas, J. (1964). Cacao and its allies a taxonomic revision of genus Theobroma. Bulletin of the United States National Museum, Smithsonian Institution, Washington, USA.

- Doyle, J. J. (1991). DNA protocols for plants. En G. Hewitt, A. W. B. Johnson, y Young, J. P. W. (Eds.), Molecular Techniques in Taxonomy (pp. 283-293). NATO ASI Series H, Cell Biology Vol. 57.

- Doyle, J. J., y Doyle, J. L. (1990). A rapid total DNA preparation procedure for fresh plant tissue. Focus, 12, 13-15.

- Girón, C., Tortolero, J., y Sánchez, P. (2007). Theobroma cacao L. (Sterculiaceae) en la región nororiental de la Isla Margarita, Estado Nueva Esparta, Venezuela. PGR NEWSLETTER-FAO-IPGRI, 138, 1-4.

- Liu, J., y Muse, S. V. (2005). Power Marker: Integrated analysis environment for genetic marker data. Bioinformatics, 21(9), 2128-2129.

- Marcano. M., Morales, S., Hoyer, M. T., Courtois, B., y Risterucci, A. M. (2009). A genomewide admixture mapping study for yield factors and morphological traits in a cultivated coca (Theobroma cacao L.) population. Tree Genetics and Genomes, 5(2), 329-337.

- Márquez, M. P. (2003). Caracterización Molecular y Morfológica de progenies de árboles plus seleccionadas dentro del "Ensayo de procedencias y progenies de Cordia alliodora" de Cenicafé-Colombia. Trabajo de Grado para optar al título de Magíster en Agricultura Ecológica, Centro Agronómico Tropical de investigadores CATIE, Escuela de Postgrado, Turrialba, Costa Rica. 80p.

- McCouch, S. R. (2002). A Laboratory Manual. Rice Laboratory, Department of Plant Breeding, Cornell University, 240 Emerson Hall, Ithaca, NY 14853, USA.

- Quiroz, J. G. (2002). Caracterización molecular y morfológica de genotipos superiores con características de cacao nacional (Theobroma cacao L.) de Ecuador. Trabajo de Grado para optar al título de Magíster Scientiae, Centro Agronómico Tropical de Investigación y Enseñanza CATIE, Programa de Educación para el Desarrollo y la Conservación, Escuela de Postgrado, Turrialba. Costa Rica. pp. 1-132.

- Sneath, P. H. A., y Sokal, R. R. (1973). Numerical Taxonomy: the principles and practice of numerical classification. San Francisco: Freeman. 573p.

- Palacio, D. (2007). Caracterización (fingerprinting) de clones de Theobroma cacao L., de interés comercial de la zona cafetera marginal baja (ZCMB) utilizando marcadores tipo fAFLP. Trabajo de Grado para optar al título de Magíster en Ciencias Biológicas, Área Biología Molecular de Plantas, Departamento de Ciencias Biológicas, Facultad de Ciencias Universidad de los Andes, Bogotá. pp. 1-104.

- Tolares, S., Marcucci, P., y Harrond, L. (2005). Identificación genética de clones en Eucalyptus grandis utilizando Microsatélites. Instituto de Recursos Biológicos CNIA, INTA Castelar.

- Virk, P., Ford-Lloyd, B., Jackson, M., y Newbury, J. (1995). Use of RAPD for the study of diversity within plant germplasm collections. Heredity, 74, 170-179.

1. Estudiante de biología en trabajo de grado, pasante año 2008 dentro del grupo de investigación en genética, biodiversidad y fitomejoramiento (GEBIOME), Universidad de Caldas, Manizales.

2. Licenciada en Biología y Química, Magíster en Biología Vegetal. Profesora ocasional Universidad de Caldas. 
3. Licenciado en Biología y Química, Magíster en Biología. Profesor de Carrera. Investigador del grupo de investigación en genética, biodiversidad y fitomejoramiento (GEBIOME), Departamento de Ciencias Biológicas, Universidad de Caldas.

4. Licenciado en Biología y Química, Magíster en Biología (Genética), Doctor en Ciencias Agropecuarias. Profesor de Carrera. Investigador del grupo de investigación en genética, biodiversidad y fitomejoramiento (GEBIOME), Departamento de Ciencias Biológicas, Universidad de Caldas. 